\title{
Investigation of Quasi Periodic Signals of X-Ray Bursts from Neutron Star AXP 1E2259+586 Using Fourier Analysis Techniques
}

\author{
S. Ally ${ }^{1}$ and N.T. Jiwaji ${ }^{2}$ \\ ${ }^{1}$ ICT Department, The Faculty of Science, Technology and Environment Studies \\ (FSTES), The Open University of Tanzania, said.ally@out.ac.tz / \\ said.ally@hotmail.com \\ ${ }^{2}$ Physical Science Department, The Faculty of Science, Technology and Environment \\ Studies (FSTES), The Open University of Tanzania, \\ noorali.jiwaji@out.ac.tz/ntjiwaji@yahoo.com
}

\begin{abstract}
Investigation of Quasi Periodic Oscillations (QPOs) in the spacecraft instrument signals provides significant insights towards understanding the physical processes that are taking place in the emissions of X-rays. The QPOs are the frequency oscillations which give us more and detailed information about the science of stars. Since astronomy is an observational and not experimental science; the physics of stars cannot be studied by traditional methods of changing variables. In this paper, we present how the Fourier analysis and its computational algorithm of Fast Fourier Transform (FFT) can be applied towards detection of the QPOs by generating the power-frequency curves of various $x$-ray bursts.

To undertake this study, the science of High Energy Astrophysics (HEA) has been applied in parallel with exploration of the digital observational data from Rossi $X$ ray Timing Explorer (RXTE) spacecraft databases which are freely made available from NASA \& ESA electronic archives. The sample data used are those of the neutron star Anomalous X-Ray Pulsar (AXP) 1E2259+586. The study involved intense computer programming using tools such as UBUNTU 10.04 OS, MATLAB 2010a UBUNTU compatible version for data visualization, HEASOFT-6.10 package with FTools and IDL software for processing of the science events.

A timing analysis of QPOs has been accomplished through applying computational technique of FFT algorithm.

The study reveals that FFT is a strong mathematical tool in finding QPOs of the $X$ ray bursts. The FFT applied on the light curve with the highest time resolution can manage to remove the background noise and detect a QPO's signal. To detect a QPO event, the FFT must show the high power peaks above $f=100 \mathrm{~Hz}$ or through the 3 sigma level of normalized FFT plots which indicates a QPO when its power level is of high value. The statistical significance of QPOs can be established by fitting with a Gaussian function to determine the width and central frequency and to assess the Fourier Analysis method using a probabilistic approach of the Monte Carlo Simulation (MCS) by running random number of simulations in MATLAB computing language.
\end{abstract}

The main contribution of this work is to demonstrate the strength of Fourier analysis in Digital Signal Processing (DSP) and show the extent on which it can be applied in analyzing spacecraft data. The software and technical challenges have been raised to promote support for researchers in developing countries.

Keywords: Fourier Analysis, FFT, QPO,X-Ray:burst,Star: neutron, Pulsar: AXP 1E2259+586 


\section{Introduction}

Investigation of Quasi Periodic Oscillations (QPOs) in the spacecraft signals provides significant insights towards understanding the physical processes that are taking place in the emissions of X-rays. The X-Ray is an electromagnetic radiation of extremely short wavelength (100 to 0.001 nanometres) produced by the deceleration of charged particles or the very high energy transitions of electrons in atoms and exhibit phenomena associated with waves that travel at the speed of light, but experiments indicate that they can also behave as particles called photons ${ }^{1}$. On the electromagnetic spectrum, they lie between ultraviolet radiation and gamma rays. They are highly ionizing and extremely harmful to life. They were discovered in 1895 by Wilhelm Conrad Rontgen, who named them X-rays for their unknown nature. The $\mathrm{X}$-ray burst is a celestial phenomena characterized by the emission of very powerful bursts of x-radiation in cycles lasting from a few seconds to a few minutes. These bursts cannot be observed on earth's surface because our atmosphere is opaque to X-rays.

The QPOs are of unique significance and are very useful in understanding the nature and physical processes happening in the stars. The Quasi Periodic Signals are the variations in the intensity of X-radiation from sources that show periodic behavior for short time intervals, and a variety of periods. The QPOs (almost, but not quite periodic) are emissions of radiation at irregular intervals due to catastrophic (e.g. earthquake type) events that happen on a neutron star. Each such event can produce a burst of energy with oscillations that are characteristic of a particular type of catastrophic event on that star. Many such bursts from different or similar events can occur, and at irregular intervals. These radiation emissions are over and above the normal emissions from the star, which are part of the background noise coming from the star.

By QPO we mean the manner in which the X-ray light from an astronomical object flickers about certain frequencies i.e. the $\mathrm{x}$ rays are emitted near the inner edge of an accretion disk in which gas swirls onto a compact object such as a white dwarf, neutron star, or black hole. The QPO phenomenon help astronomers understand the innermost regions of accretion disks and the masses, radii, and spin periods of white dwarfs, neutron stars, and black holes.

In this study, we present the QPOs observed from the Neutron Star called Anomalous X-ray Pulsars (AXPs) which are believed to be magnetars, i.e., neutron stars with an intense magnetic field of the order of 1014 to 1015 G (Duncan and Thompson, 1992; Woods and Thompson, 2006).

The Neutron Stars (NS) are very dense objects that mark the end

${ }^{1}$ X-ray definition, http://www.answers.com/topic/x-ray, [Accessed 15

April 2014] 
points of the lives of some stars. Neutron stars are compact objects categorized as rotationally powered having radio pulsars $>1000$, accretion powered having X-ray binary $>1000$ and magnetically powered having Soft Gamma Repeaters (SGRs) and Anomalous X-ray Pulsars (AXPs) $>10$. The NS are final products of stellar evolution and are born in supernova explosions after their presupernova progenitors (giant or supergiant stars) exhaust nuclear fuel in their cores ${ }^{2}$. Neutron stars are very hot and are supported against further collapse because of the Pauli Exclusion Principle which states that no two neutrons can occupy the same quantum state simultaneously. The whole event is usually referred to as a core-collapse type II supernova explosion. The NS have typical masses $M \approx 1.4 M_{0}$ and extremely small radii $R \approx 10 \mathrm{~km}$, thus their masses are close to the solar mass $M_{0} \approx 1.4989 \times 10^{33} \mathrm{~g}$, but their radii are 10 times smaller than the solar radius $R=6.96 \times 10^{5} \mathrm{~km}$. According to current theories, a NS can be subdivided into the atmosphere and four main internal regions: the outer crust, the inner crust, the outer core, and the inner core as shown in figure.

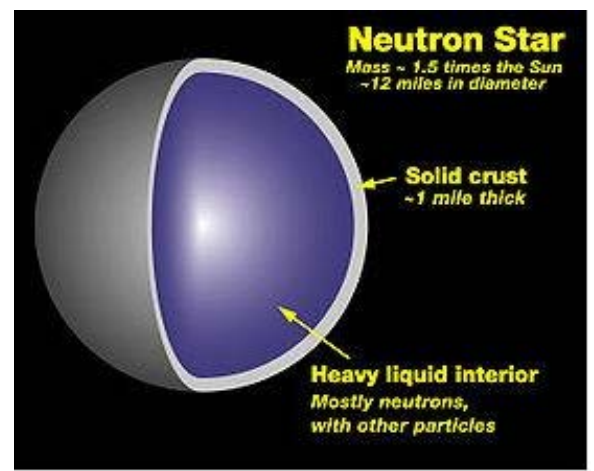

Figure 1(a): Structure of Neutron $\operatorname{Star}^{3}$

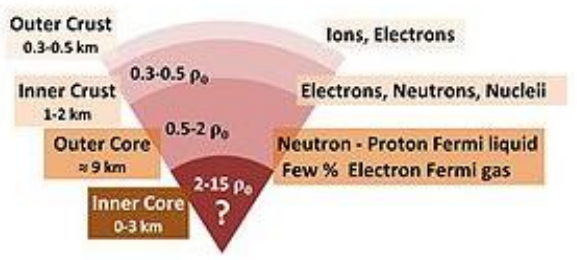

Figure 1(b): Structure of Neutron Star-Compositon of layers ${ }^{3}$.

Current understanding of the structure of neutron stars is defined by existing mathematical models. Neutron stars rotate extremely rapidly after their creation due to the conservation of angular

2 Neutron Star definition, http://www.astro.umd.edu/miller/nstar.html, [Accessed 23 June 2014]

${ }^{3}$ Structure of the Neutron Star:

http://commons.wikimedia.org/wiki/File:Neutron_star_cross_section.jpg, [Accessed 26 May 2014] 
momentum. The most rapidly rotating neutron star currently known, PSR J1748-2446ad, rotates at 716 revolutions per second. At present there are about 2000 NSs in the Milky Way and the Magellanic Clouds, the majority of which have been detected as radio pulsars. NS are born with high speeds $400 \mathrm{~km} / \mathrm{s}$. One of the closest known NS is PSR J0108-1431 at a distance of about 130 parsecs (or 424 light years).

The AXPs are believed to be magnetars. The magnetars are characterized by the repetitive emission of bright bursts of lowenergy gamma rays with short durations $(\sim 0.1 \mathrm{~s})$ and are also considered to be persistent X-ray pulsars with slow spin periods of approximately from 2 to $12 \mathrm{~s}$ and rapid spin-down rate of $1 \mathrm{~ms} / \mathrm{yr}$.

At present, there are 18 magnetars of which 8 are SGRs and 10 are AXPs (Mereghetti, 2008). Based on the magnetar model, the dominant source of energy of magnetars is their intense magnetic field. The persistent X-ray emission is attributed to magnetospheric currents driven by twists in the magnetic field. The detection of QPOs in magnetars has triggered a boost in the theoretical modeling that included attempts to construct more refined models that consider crust/core coupling, magnetic field geometry, elastic properties of the crust, and other effects (ElMezeini, et al., 2010).

By their very nature, QPOs show non-periodic noise that appears as a continuous curve in the power spectrum; hence its investigation is not straight forward. Since QPOs are frequency oscillations which give us more and detailed information about the science of stars, its investigation cannot be studied using experimental methods or methods of changing variables. Unlike statistics or business applications time series which have records with equal intervals, the astronomical time series are complex due to gapped data streams (diurnal and monthly cycles, satellite orbital cycles and telescope allocations), heteroscedastic measurement errors (signal-to-noise ratio differs from point to point) and Poisson processes (individual photon/particle events in high energy astronomy). Astronomical time series are somewhat different from the standard time series often used in other branches of science and businesses. The random, often sparse, gapped and unknown nature of astronomical observational sequences makes most of techniques of the standard time series analysis unusable.

To understand the QPOs accurately and be able to detect changes that occur in the signals (significant bursts) against background noise due to the dynamical processes happening in the star, a mathematical approach of Fourier analysis needs to be applied. In this research, the astronomical time series are expressed in form of light curves which are of sine, impulse-like, eclipse, complex and multi-periodicity time series. The challenge is to convert the digital data collected from spacecrafts collected over long periods 
of time to produce a frequency distribution of the energy from each burst and hence produce its intensity spectrum. Such a spectrum gives a picture of the actual characteristics of the radiation from the burst, which is of interest to astrophysicists who can interpret the spectrum to understand the physical processes taking place in the star using physics theories.

We present how the Fourier analysis and its computational algorithm of Fast Fourier Transform (FFT) can be applied towards detection of the QPOs by generating the power-frequency curves of various $\mathrm{X}$-ray bursts.

\section{Fourier Analysis Technique}

Fourier analysis is mathematical technique that is useful in the study of signals of periodic nature i.e. signal that repeat itself after a certain amount of time (Techtarget, 2014) such that $f(t+T)=f(t)$ for some $T$ and all $t$. The period is the smallest value of $T$ which can take value $2 T, 3 T, 4 T, \ldots$ Geometrically, a periodic function has graph which exhibits translational symmetry i.e. the graph of $f$ is invariant under translation in the x-direction by a distance of $T$. A Fourier analysis defines periodic waveforms in terms of trigonometric functions ${ }^{4}$. A Fourier series is a way of expressing a wave function (usually amplitude, frequency, or phase versus time) as a sum of sine and cosine functions. It is uniquely defined by constants known as Fourier coefficients. Van de Kliss (1989) has represented the coefficients by $a_{0}, a_{1}, a_{2}, a_{3}$, $\ldots, a_{n}, \ldots$ and $b_{0}, b_{1}, b_{2}, b_{3}, \ldots, b_{n}, \ldots$ in a Fourier series $F(x)$ where $x$ is an independent variable (usually time) with the following form:

$$
\begin{aligned}
& F(x)=\frac{a_{0}}{2}+a_{1} \cos (x)+b_{1} \sin (x)+a_{2} \cos (2 x)+ \\
& b_{2} \sin (2 x)+\ldots+a_{n} \cos (n x)+b_{n} \sin (n x)+\ldots \\
& \left.f(x)=a_{0}+\sum_{n=1}^{\infty}\left(a_{n} \cos (n x)+b_{n} \sin (n x)\right) \quad \text { for }-\pi \leq x \leq \pi \quad \text { (Eq. } 2\right)
\end{aligned}
$$

The aim is always to find formula for the coefficients $a_{n}$, and $b_{n}$, in terms of $f(x)$. If we integrate both sides of Eq.2 we get

4 Periodic Signals definition: http://en.wikibooks.org/wiki/Signals-andSystems/Periodic-Signals, [Accessed 09 March 2014] 


$$
\begin{aligned}
& \int_{-\pi}^{\pi} f(x) d x=\int_{-\pi}^{\pi} a_{0} d x+\int_{-\pi}^{\pi} \sum_{n=1}^{\infty}\left(a_{n} \cos (n x)+b_{n} \sin (n x)\right) d x \\
& =2 \pi a_{0}+\sum_{n=1}^{\infty} a_{n} \int_{-\pi}^{\pi} \cos (n x) d x+\sum_{n=1}^{\infty} a_{n} \int_{-\pi}^{\pi} \sin (n x) d x
\end{aligned}
$$

But $\left.\int_{-\pi}^{\pi} \cos (n x) d x=\frac{1}{n} \sin n x=\frac{1}{n}[\sin n \pi-\sin (-n \pi)]=0\right]$ because $n$ is an integer. Similarly, $\int_{-\pi}^{\pi} \sin (n x) d x=0$. So, $\int_{-\pi}^{\pi} f(x)=2 \pi a_{0}$ and solving for $a_{0}$ gives $a_{0}=\frac{1}{2 \pi} \int_{-\pi}^{\pi} f(x) d x$.

To determine $a_{n}$ for $n \geq 1$ we multiply both sides of the equation 2 by $\cos m x$ where $m$ is an integer and $m \geq 1$ and integrate term by term from $-\pi$ to $\pi$.

$$
\begin{aligned}
& \left.\int_{-\pi}^{\pi} \cos m x d x=\int_{-\pi}^{\pi}\left(a_{0}+\sum_{n=1}^{\infty} a_{n} \cos n x+b_{n} \sin n x\right)\right) \cos m x d x \\
& =a_{0} \int_{-\pi}^{\pi} \cos (m x) d x+\sum_{n=1}^{\infty} a_{n}+\int_{-\pi}^{\pi} \cos n x \cos m x d x+\sum_{n=1}^{\infty} b_{n} \int_{-\pi}^{\pi} \sin n x \cos m x d x
\end{aligned}
$$

Note that $\int_{-\pi}^{\pi} \sin n x \cos m x d x=0$ for all $n$ and $m$ and

$$
\int_{-\pi}^{\pi} \cos n x \cos m x d x= \begin{cases}0, & n \neq m \\ \pi, & n=m\end{cases}
$$

so, the only non zero term in the equation is $a_{m} \pi$ and we get

$\int_{-\pi}^{\pi} f(x) \cos (m x) d x=a_{m} \pi$. Solving for $a_{m} n$ and then replacing $m$

by $n$ we have $a_{n}=\frac{1}{\pi} \int_{-\pi}^{\pi} f(x) \cos n x d x$ for $\mathrm{n}=1,2,3, \ldots$

Similarly if we multiply both sides of equation 2 by $\sin m x$ and integrate from $-\pi$ to $\pi$, we get $b_{n}=\frac{1}{\pi} \int_{-\pi}^{\pi} f(x) \sin (n x) d x$ for $n=1,2,3, \ldots$

(Eq.

5)

\section{Research Methodology}

To undertake this study, the science of High Energy Astrophysics (HEA) has been applied in parallel with exploration of the digital observational data from Rossi X-ray Timing Explorer (RXTE) spacecraft databases which are freely made available from NASA 
\& ESA electronic archives. The RXTE ${ }^{5}$ is a satellite that observes the time structure of astronomical X-ray sources with three inbuilt instruments which are Proportional Counter Array (PCA), HighEnergy X-ray Timing Experiment (HEXTE), and All Sky Monitor (ASM). The satellite was launched on December 30, 1995 and has a time scales from microseconds to months and posses energy ranging from 2 to $250 \mathrm{keV}$.

The sample data used are those of the neutron star Anomalous XRay Pulsar (AXP) 1E2259+586. To assemble and analyze data, serious computer programming skills required. We used UBUNTU 10.04 OS, MATLAB 2010a version which is UBUNTU $^{6}$ compatible for data visualization, HEASOFT-6.10 package $^{7}$ (HEASOFT, 2013) with FTools and IDL software for processing of the science events.

A timing analysis of QPOs has been accomplished through applying computational technique of FFT algorithm. The HEASOFT package was very useful in downloading and conversion of the original tar files into light curves and MATLAB files.

\section{Data Analysis and Programming}

To determine the characteristics of the periodicity in observed signals, an FFT computational algorithm has been used to generate power frequency curves. Since the space signals come in digital form and are discrete and periodic, the Discrete Fourier Transform (DFT) fit the purpose. The DFT is the only type of Fourier transform that can be used in Digital Signal Processing taking advantage of the sinusoids instead of the square or triangular waves. The FFT is DFT technique which is an ingenious algorithm that decomposes a DFT with $\mathrm{N}$ points, into $\mathrm{N}$ DFTs each with a single point, so FFT is typically hundreds of times faster than the other methods of DFT such as simultaneous equations/Gauss elimination and Correlation. Additionally, FFT is capable of accepting huge amount of data points.

The following is a MATLAB code that opens the FITS file into the light curves which displays the count rate (counts/sec) and time (sec) variables.

5 RXTE Space Mission: http://heasarc.nasa.gov/docs/xte/rxte.html

[Accessed 05 February 2013]

${ }^{6}$ UBUNTU Operating System:

http://www.ubuntu.com/desktop/get-ubuntu/download,

[Accessed 22 January 2013]

${ }^{7}$ http://heasarc.nasa.gov/lheasoft/download.html, [Accessed 11 April 2013] 
MATLAB openlc function

function $[\mathrm{r}, \mathrm{t}]=$ openlc(FileName,PlotIt)

clearrt;

\%FileName=input('Enter The Light Curve (FITS) file name: ');

FITSData=fitsread(FileName,'BinTable');

FITSFileInfo=fitsinfo(FileName)

PrimaryData=FITSFileInfo.PrimaryData;

BinaryTable=FITSFileInfo.BinaryTable;

t=FITSData $\{1\}-$ FITSData $\{1\}(1)$;

$\mathrm{r}=$ FITSData $\{2\}$;

$\mathrm{w}=$ find $(\mathrm{r}<0)$;

$r(w)=\operatorname{abs}(r(w))$;

if $(\bmod ($ length $(\mathrm{t}), 2)) \sim=0$

$\mathrm{t}(\mathrm{end})=[] ;$

$\mathrm{r}(\mathrm{end})=[]$;

end

ifPlotIt $>=0$

figure; stairs(t,r);

xlabel('Time (s)'); ylabel('Count Rate (Counts / s)');

\%plot(t,r,'o')

end;

\section{MATLAB Commands \& Output}

filename='FS3f_56b64b0-56b722e-obs2-ev2-Burst01-0.1ms-lc.lc' filename =FS3f_56b64b0-56b722e-obs2-ev2-Burst01-0.1ms-lc.lc

$>>[\mathrm{r}, \mathrm{t}]=$ openlc(filename, 1$)$;

FITSFileInfo $=$

Filename: 'FS3f_56b64b0-56b722e-obs2-ev2-Burst01-0.1ms-lc.lc'

FileModDate: '13-Apr-2014 13:21:10'

FileSize: 43200

Contents: \{'Primary' 'Binary Table' 'Binary Table'\}

PrimaryData: [1x1 struct]

BinaryTable: [1x2 struct]

The following is a MATLAB code that creates the corresponding powerfrequency curves with power and frequency variables.

function $[\mathrm{P}, \mathrm{f}, \mathrm{df}, \mathrm{fs}, \mathrm{N}, \mathrm{dt}]=$ powerfreq(r,t,title)

$\mathrm{N}=$ length(r); \% Number of bins in the burst

$\mathrm{dt}=\mathrm{t}(2)-\mathrm{t}(1)$;

$\mathrm{fs}=1 . / \mathrm{dt}$;

$\mathrm{df}=1 . /(\mathrm{dt} * \mathrm{~N})$;

$\mathrm{f}=(0: \mathrm{df}:(\mathrm{fs}-\mathrm{df}))$ '; \%frequency

$\mathrm{P}=2 *(\operatorname{abs}(\mathrm{fft}(\mathrm{r}))) . \wedge 2 . / \operatorname{sum}(\mathrm{r}) ; \%$ Power

figure; stairs(f,P); ylabel(title);

\section{MATLAB Commands}

$>>\operatorname{load}($ 'exampleLC.mat')

>> $[\mathrm{P}, \mathrm{f}, \mathrm{df}, \mathrm{fs}, \mathrm{N}, \mathrm{dt}]=$ powerfreq(r,t,'Power vs Frequency')

\section{Using Fast Fourier Transform (FFT) to detect QPOs}

A QPO is identified by performing power spectrum of the time series of the X-rays, which is possible only through applying Fourier analysis that can represent signals as a periodic sine and 
cosine functions. Because these data are digital and hence of discrete type, and are time series data, a computational algorithm of Fast Fourier Transform (FFT) can be used to understand their frequency distributions which are our main interest. Radiation originating from $\mathrm{X}$-ray sources possesses high frequencies and therefore emits high energies, so by performing Fourier analysis and plotting their frequency distributions, we can filter out the background noise from the significant bursts.

The FFT is a suitable computational algorithm for understanding QPOs in the study X-Ray timing and analysis of signals in the frequency domain. Once we know the Fourier coefficients, we have entered the time series into its different frequency components and have entered the frequency domain. Since FFT algorithm involves both the window length (i.e. the length of the input data vector) and the transform length (i.e. the length of the output - the computed DFT). The execution time of an FFT algorithm depends on the transform length. It is fastest when the transform length is a power of two, and almost as fast when the transform length has only small prime factors.

A QPO is a sloppy oscillation which can be due to intrinsic frequency variations, finite lifetime and amplitude modulation and are classified as Normal QPOs, High Frequency QPOs and Burst Oscillations. They are observed from the sources as either Z-Shape or Atoll Shaped which is also called Banana shaped (Arzoumanian Z., 2003).

Normal QPOs have low frequencies of up to $100 \mathrm{~Hz}$ while High Frequency QPOs often have twin peaks (also called kHz QPOs). On the other hand, burst oscillations do not occur in every burst; they result from anisotropic burning on the surface and rotation of neutron star. They are very close to neutron star rotation frequency.

The sources where QPOs are observed from are the Low Mass XRay Binaries which include Neutron Stars, X-Ray bursters and Black Hole Candidates. In RXTE, the QPOs are detected as COUNTS.

The basic assumptions for detection of the QPOs include:

(i). The detected QPO's are found in bursts with different durations, morphologies and brightness.

(ii). Real signals are NOT continuous.

(iii). Real Signals are NOT infinitely extended.

(iv). No signals are introduced by instrument (read time), dead time, orbit of spacecraft and rotation period of Earth (and harmonics).

\section{Results}


After running a source data file (science event gx0), the following light curve was generated.

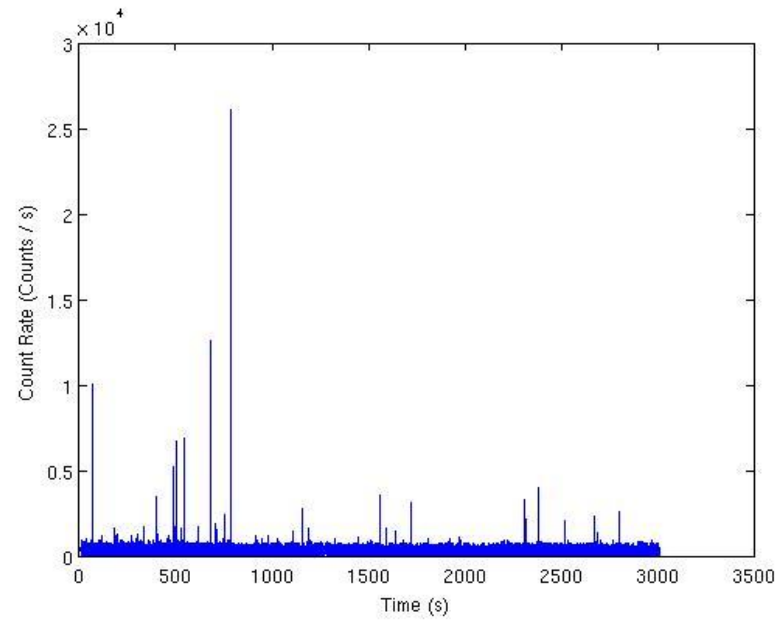

Figure 2: The Light Curve for the Science Event gx0 - the 16ms Burst

In the light curve, 10 significant bursts (with highest peaks) were studied. Then the light curve for the first brightest burst was generated as shown in the figure 4 for $1 \mathrm{~ms}$ bin size.

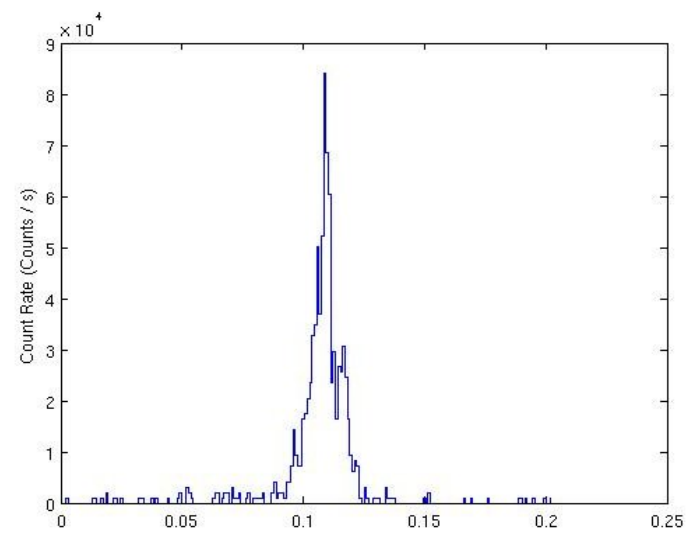

Figure 3: The Light Curve for the Science Event gx0 - First Brightest Burst

The detailed graph of Figure 5 is obtained when the burst shown in figure 4 is restricted on the $\mathrm{X}$-axis for the time that the burst was happening for the chosen resolution of $1 \mathrm{~ms}$ (i.e. bin size of $0.00097656250 \mathrm{~s}$ ) and initial time (MET for RXTE) of $2.6703330 \mathrm{e}+08 \mathrm{~s}$. 


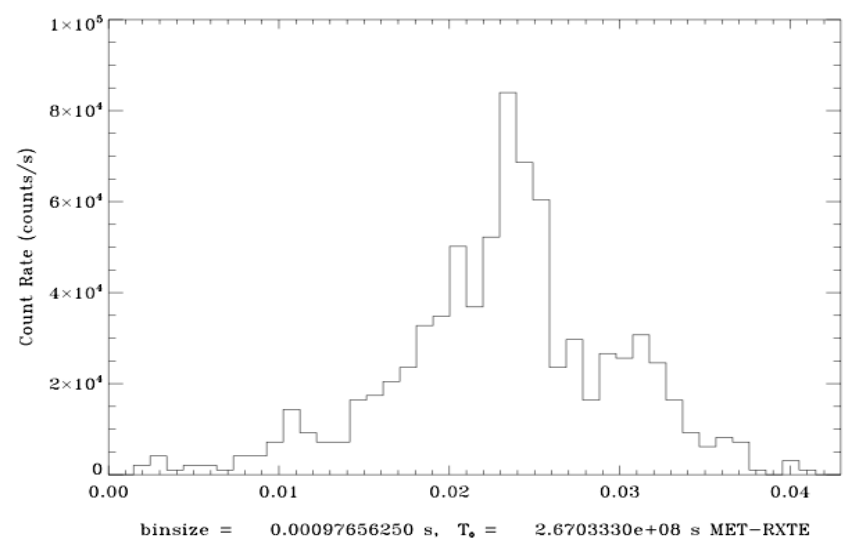

Figure 4: Detailed First Brightest Burst Light Curve for Science Event gx0

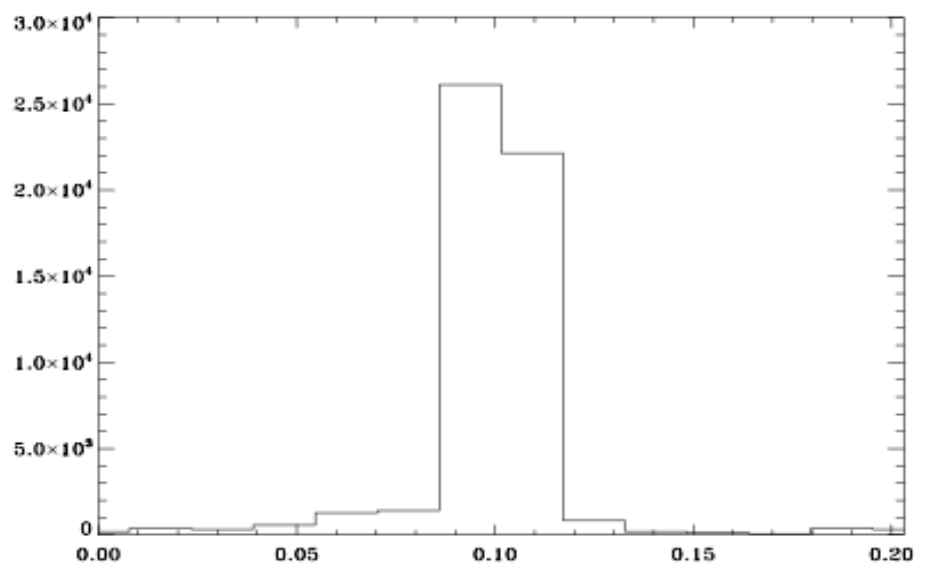

Figure 5: The Light Curve for the Science Event gx0 - the Brightest Burst 16ms

Then the FFT executed and the power frequency curve for the brightest burst generated.

\subsection{Observations from the X-Ray Bursts with $16 \mathrm{~ms}$ bin $\mathrm{s}$}

The following table shows results obtained after loading a MATLAB file 'event-gx0-lc-16ms.mat' and running a power frequency using function:

$[P, f, d f, f s, N, d t]=$ powerfreq(r,t,'Power vs. Frequency')

This file is a result of a burst with bin size of $16 \mathrm{~ms}$

In figure 6, the X-range has been cut to a half and zoomed along the $\mathrm{y}$-axis and $\mathrm{x}$-axis to find the 10 brightest bursts for filtering the background. Another version of the light curve was produced at the highest possible time resolution, i.e. 125 micro seconds. 


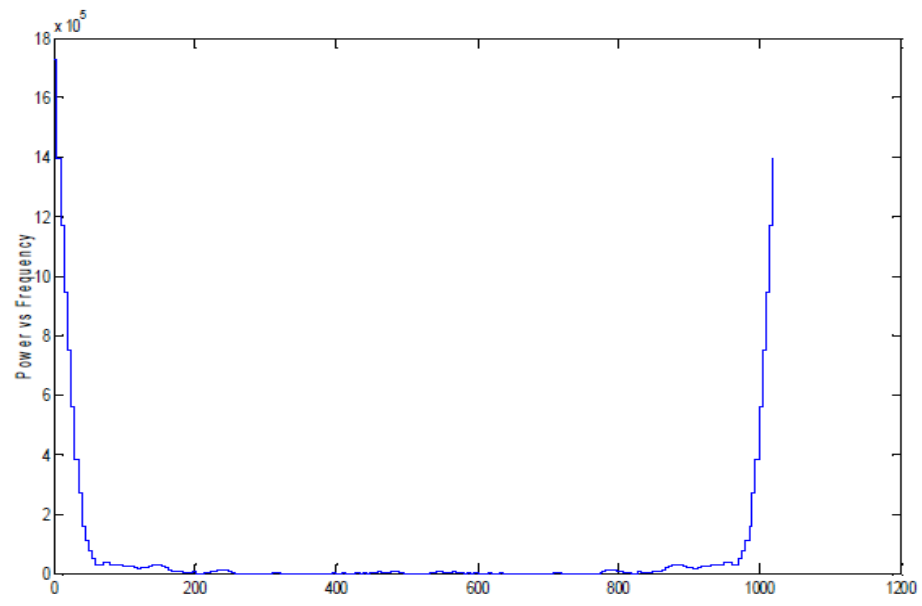

Figure 6: The Power-Frequency Diagram for the Science Event gx0 - the Brightest Burst

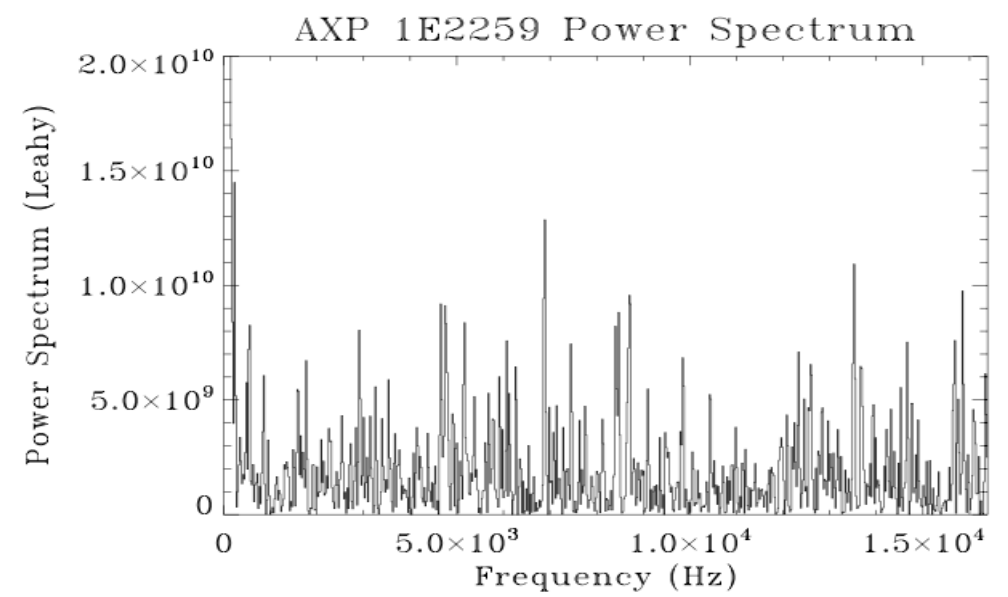

Figure 7: The Light Curve for the Science Event gx0 - the Brightest Burst 32us

Due to technicalities of software limitations, by running FFT on the light curve at the highest resolution we were not able to detect the QPOs at given frequencies.

However, the FFT of the bursts that show QPOs must have their power (y-axis) at the range of 0 to 50. Otherwise, for the bursts which have the power outside this range, their powers must be normalized.

Consequently, the 3 sigma level that indicates QPO when its power level is higher than $100 \mathrm{~Hz}$ has also not been calculated. To obtain the statistical significance of a QPO, it must be determined by fitting it to Gaussian function to determine the width and central frequency.

\subsection{Assessment of the FFT Results Using Monte Carlo Simulations}

One of the scientific methods used to assess the FFT results, is 
application of the Monte Carlo Simulation (MCS). The MCS is normally applied only on the bursts that have shown QPOs in their FFT plots. If for instance, the selected bursts do not show QPOs, other bursts should be considered including the lower peaks by looking on the background level i.e. by choosing the bursts that are well above the background. And if for instance, the QPOs are not found in other bursts, we can also look at the persistent emission (background level with no bursts).

Alternatively, all bursts can be amplified together in a common signal and do one FFT on their joined light curve at the highest resolution by using "seextrct" on the event mode file (that contain all bursts) and enter the individual time ranges of each burst (zoomed) followed by a comma, i.e. in a form of $t_{1}-t_{2}, t_{2}-t_{3}$, etc. The result of this is a joined light curve at the highest time resolution that can be used with FFT. Alternatively, each burst light curve can be read and joined them in IDL in a new array. With this approach, a calibration bitfile with seextrct is used by simply tell it by typing psetseextrct and see it in the bitfile field.

The MCS is done only on the bursts that have QPOs as a way of assessing the significance of the QPOs.

\section{Findings and Discussion}

The mathematical development of the Fast Fourier Technique (FFT) has been mathematically presented. The MATLAB programming codes for analyzing spacecraft data using FFT were written and we were able to produce light curves for a science event at various resolutions. Further MATLAB codes were written to provide the power spectrum of the oscillations in a particular burst in the scientific event. Power spectra allow physical characteristics such as QPOs to be detected. Due to limitations of software bugs all bursts could not be analyzed hence QPOs in this particular scientific event could not be identified.

We have shown that the FFT is a powerful technique for analyzing huge amounts of available spacecraft data. Limitations of bugs in software prevented us from drawing out the properties of the neutron star under study. However the mathematical analytical techniques have been verified.

\section{Conclusion and Recommendations}

In the last few years there have been enormous advances in the study of QPOs from neutron stars. The high frequency QPOs were unknown and unsuspected prior to the launch of RXTE in 1995. With current data, a lot of knowledge of the inner-most part of the neutron stars is expected. The modern space signal processing applications could be impossible without an efficient method for computing DFTs. This research has analyzed how FFT can be used to understand the QPOs and method validated 
probabilistically with MCS method. The applied mathematical technique can be extended to analyze any space craft data of the same nature.

Our FFT approach was completed through MATLAB on the major $\mathrm{X}$-Ray bursts of $16 \mathrm{~ms}$. In all undertaking of this problem, the computational algorithm of the FFT had efficient timings and best fit for detecting significant bursts from QPOs. The idea behind was to separate the QPOs for different sizes of input data and compare the results using two scientific methods of FFT computation and Monte Carlo method.

We recommend that the technical capacity for such analysis should be increased and support from established researchers should be made available so that the exponentially increasing electronic data from spacecrafts are analysed speedily to understand better all the different components of our Universe.

\section{Acknowledgement}

We would like to express our sincere gratitude and many thanks to Dr. Alaa Ibrahim, Head of Physics department at the American University in Cairo for his technical guidance in undertaking this research project. This work was supported in part by the Open University of Tanzania. The research has made use of data obtained through the High Energy Astrophysics Science Archive Research Center Online Service, provided by the NASA Goddard Space Flight Center.

\section{References}

Arzoumanian Z., (2003), The Why \& How of X-Ray Timing. (USRA/NASA-GSFC), lecture notes.

Bracewell, R. (1965). The Fourier Transform and its Applications (Mc-Graw Hill); $3^{\text {rd }}$ edition, ISBN 9780071160438 , Prentice-Hall

Duncan, R.C., and Thompson, C. (1992). Formation of very strongly magnetized neutron stars - Implications for gamma-ray bursts. Astrophysical Journal, 392, L9

El-Mezeini A. M. \& Ibrahim A. I., (2010), Discovery of QuasiPeriodic Oscillations in the Recurrent Burst Emission from SGR 1806-20; The Astronomical Journal Letters, Volume 721, Number 2

Mereghetti S., et al., (2008). The XMM-Newton view of magnetars. Willey Online Library. 
Strohmayer T. E., \& Watts A. L., (2005). Discovery of Fast XRay Oscillations during the 1998 Giant Flare from SGR 1900+14. The Astronomical Journal Letters, 632(2)

Van der Klis M., (1989). Fourier Techniques in X-Ray Timing; NATO ASI Series, 262, 27-69

Watts A. L., \& Strohmayer T. E., (2006). Detection with RHESSI of High-Frequency X-Ray Oscillations in the tail of the 2004 Hyperflare from SGR 1806-20. Astrophysical Journal, 637, L117-L120

Woods, P.M., \& Thompson, C., (2004). Soft Gamma Repeaters and Anomalous X-ray Pulsars: Magnetar Candidates. Astrophysical Journal, 608, L49-L52, astro-ph/0401561

Woods P. M., Fotis P. G., Kaspi V. M., (2004). A Comprehensive Study of the X-Ray Bursts from the Magnetar Candidate $1 \mathrm{E}$ 